\title{
Défis actuels et à venir en matière d'analyse de traces et d'ultra-traces
}

\author{
P. Toulhoat ${ }^{1}$ \\ 1 CEA Saclay \& CNRS, Institut des sciences analytiques, université Claude-Bernard Lyon 1 , \\ 43, avenue du 11 -Novembre-1918, 69622 Villeurbanne Cedex - France \\ e-mail: pierre.toulhoa@@univ-lyon 1.fr
}

\begin{abstract}
Résumé - L'analyse de traces et d'ultra-traces constitue depuis de longues années un moteur de progrès important en chimie analytique. La chimie environnementale, la radiochimie, la biologie, la santé, l'agroalimentaire sont des prescripteurs d'analyses de traces, avec des exigences qui s'accroissent sans arrêt : abaisser les limites de détection, tout en diminuant le coût et le temps de réponse, améliorer la qualité de l'information analytique. On voit ainsi apparaître des demandes relatives à la nature chimique précise de l'analyste et de son environnement chimique proche. Ces informations, qui vont au-delà d'un simple chiffre assorti de son intervalle de confiance, sont essentielles à la construction des modèles, permettant de comprendre les systèmes étudiés, et d'en prévoir l'évolution. À partir de l'étude de cas de contamination environnementale, on peut entrevoir les différents volets d'un problème, auxquels correspondent des exigences et donc des méthodes analytiques différentes. La détection d'ultra-traces d'actinides et de produits de fission fait l'objet d'un renouveau d'intérêt, suscitant de nouveaux développements technologiques. L'étude de la spéciation de ces mêmes éléments dans les milieux géologiques et biologiques est essentielle pour permettre d'évaluer la sûreté des méthodes de gestion des déchets nucléaires. De nombreuses techniques permettent maintenant d'aborder ces problèmes, tant dans des matrices liquides que solides, et ce, au niveau des traces, avec parfois une résolution spatiale impressionnante. Enfin, l'irruption des micro- ou nanotechnologies en matière d'analyse permet d'entrevoir une nouvelle révolution.
\end{abstract}

\begin{abstract}
Present and Future Challenges in Trace and Ultra-Trace Analysis - The analysis of trace and ultra-trace elements is continuously stimulating the progress in analytical chemistry. Environmental chemistry, radiochemistry, biology, health, agri-food are prescriptors of trace analyses, with continuously increasing exigencies: lowering detection limits, lowering costs and analysis time, improving the quality of analytical information. Precise data about the chemical identity and chemical environment of analytes are now requested. Such pieces of information, beyond simple numerical data and confidence intervals, are necessary to understand studied systems, and to predict their evolution. From environmental contamination cases, one can envisage the various aspects of a problem, with for each of them its own exigencies and specificities in terms of analytical methods and approaches. The detection of traces and ultra-traces of actinides and fission products has been recently revisited and stimulates new technological developments (non proliferation issues, waste management). Data on their speciation in geological and biological media are essential for evaluating the safety of nuclear waste repositories. Various techniques are now used to determine speciation in liquid samples or on surfaces, with tremendous spatial resolutions or sensitivities. A new revolution in analytical chemistry is expected with the development of micro- or nano-analytical technologies.
\end{abstract}




\section{INTRODUCTION}

La recherche de la performance en matière de limite de détection a caractérisé la chimie analytique et l'instrumentation, et ce principalement entre la fin des années 1960 et la fin des années 1980. Les années 1990 ont vu l'arrivée en masse dans les laboratoires d'appareils de plus en plus compacts et performants comme les ICP-MS. Bien souvent, ces appareils ne sont pas utilisés au plus poussé de leur limites instrumentales, car les utilisateurs doivent faire face à de nombreux problèmes, allant de la représentativité de l'échantillon à la maîtrise des blancs et artefacts au long de la chaîne d'analyse. Par ailleurs, l'exploitation de ces résultats est bien souvent incomplète, voire inexistante.

En conséquence, il faut donc rappeler la maxime : «À chaque problème sa solution». L'étude d'un cas type en chimie environnementale permet de bien poser les questions et de déterminer le type de réponse analytique appropriée (fig. 1). L'exemple de la pollution accidentelle du bassin hydrographique de la rivière Tisza, affluent du Danube, en 2000 peut servir de guide. L'exploitation de l'or par cyanuration de résidus miniers dans la région de Baia Mare (Roumanie) s'est développée de manière intensive. À la suite de fortes précipitations, une digue d'un bassin de rétention s'est rompue, entraînant une pollution importante par le cyanure et de nombreux métaux lourds qui s'est répandue sur plusieurs dizaines de kilomètres en aval [1]. Des effets immédiats tels que la mortalité élevée des poissons, la disparition du plancton, etc., ont été ressentis. Le panache de pollution s'est dispersé, et la vie a petit à petit repris son cours. Pour autant, les effets à long terme (diminution de la biodiversité, atteinte durable des écosystèmes) sont encore très mal appréciés. Le transport puis le métabolisme du cyanure, et des métaux auxquels il peut être associé sous forme de complexes, est très incomplètement connu. Au-delà de ces questions scientifiques encore non résolues, il est possible de voir sur un tel cas comment se positionnent les différentes demandes en matière d'analyse.

\section{Surveillance, détection, alerte}

La rapidité d'exécution de l'analyse est ici primordiale. Des laboratoires de proximité doivent pouvoir procéder à des analyses, parfois semi-quantitatives, afin de vérifier le bon fonctionnement d'un système ou la qualité environnementale d'un milieu. Parfois, ces analyses relèvent du domaine des traces (métaux lourds), compte tenu des normes et seuils parfois très bas. C'est dans ce contexte que des réseaux de capteurs automatisables prennent tout leur sens et font l'objet de développement et d'expérimentations. Les concentrations limites mesurables par des capteurs atteignent maintenant des valeurs en dessous de $10^{-7} \mathrm{M}$ à $10^{-9} \mathrm{M}$. Des solutions diverses sont actuellement testées : capteurs électrochimiques $[2,3]$ optiques [4], etc. Si ces systèmes sont prometteurs, ils n'ont pas tous encore franchi - loin de là - le stade du laboratoire et doivent être testés et validés sur le terrain. Une première évaluation de ce genre de méthodes avait déjà permis ce constat [5], mais il semble que le passage à des systèmes opérationnels soit encore assez rare.

\section{Évaluation quantitative de l'impact à court terme}

La mesure des concentrations précises en divers contaminants, dans l'ensemble des compartiments affectés par une pollution accidentelle ou diffuse, est un travail qui met à l'épreuve les laboratoires d'analyse. Outre les difficultés liées à l'échantillonnage ou au prélèvement, l'étape cruciale est la mise au point de méthodes normalisées, reproductibles et traçables, qui nécessitent en particulier de disposer de matériaux de référence. Pour les analyses élémentaires, la situation s'est bien améliorée, et à tous les niveaux (national, européen, international) existent des systèmes de normalisation des méthodes analytiques, d'accréditation des laboratoires. Des matériaux de référence sont distribués, pour l'analyse de traces dans des matrices aussi diverses que des végétaux, comme les choux [6], des animaux, des sols, et bien évidemment, des eaux.

\section{Évaluation des impacts à long terme}

Les effets à long terme, qu'ils soient environnementaux ou biologiques, peuvent s'étudier si l'on est capable de déterminer la forme chimique précise des contaminants (ou la répartition des différentes formes s'il en existe plusieurs). En effet, la réactivité biologique et chimique, ainsi que les modalités de transport et de transfert dépendent très étroitement de la spéciation. Cette exigence de compréhension des systèmes est à la base d'une démarche permettant de faire des prédictions à long terme. Les développements analytiques autour de la spéciation de métaux à l'état de traces ont commencé réellement au début des années 1980, puis ont eu un développement très important. De très gros progrès ont été effectués depuis, et la spéciation est devenue une branche importante de la chimie analytique environnementale. La disponibilité de matériaux de référence reste en revanche un problème très délicat [7]. Toute cette problématique peut se transposer assez facilement à des éléments radioactifs, et les recherches relatives à la spéciation des radioéléments se sont développées parallèlement à celles concernant des métaux lourds. Quelques exemples seront développés un peu plus loin.

\section{ULTRA-TRACES D'ACTINIDES ET DE PRODUITS DE FISSION}

L'analyse d'éléments ou d'isotopes à l'état de traces a constitué et constitue encore un enjeu important dans la biologie comme dans les sciences nucléaires et de l'environnement. 
Surveiller, détecter, alerter

mots clés : capteurs, analyse en ligne, analyse in situ

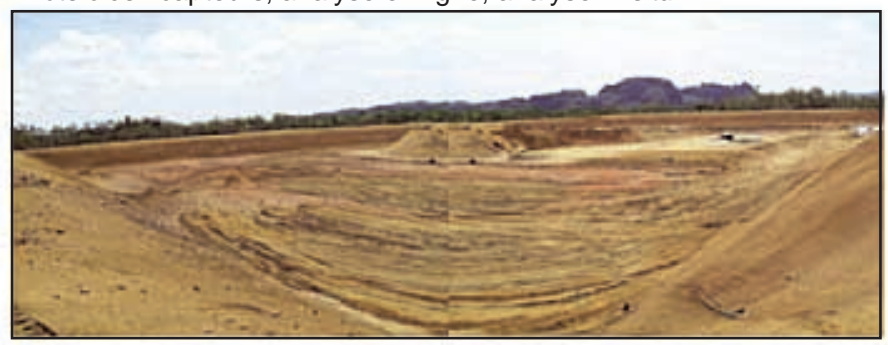

Rapidité, fiabilité, aide à la décision

Analyser quantitativement pour évaluer un impact

mots clés : traces et ultra-traces, matériaux de référence

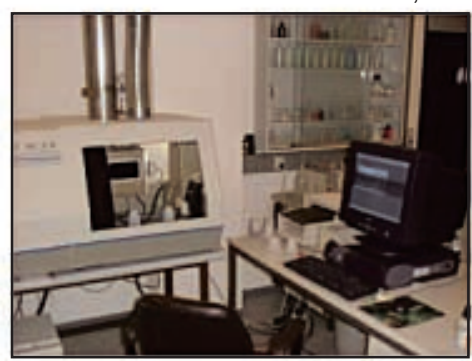

Précision, limites

de détection

Étudier les formes chimiques des contaminants pour prévoir les effets à long terme

mots clés : spéciation, couplages, analyse in vivo, modélisation

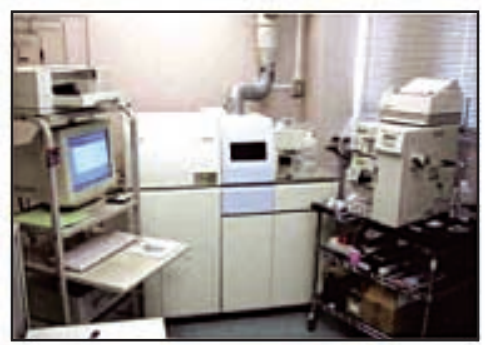

Comprendre à l'échelle moléculaire pour prévoir

Figure 1

Classes de problèmes en chimie analytique environnementale.

Classification of problems in analytical environmental chemistry.

Pendant longtemps, les isotopes radioactifs de certains éléments ont constitué les entités les plus facilement détectables à l'état de traces ou d'ultra-traces grâce aux rayonnements qu'ils émettent (gamma, alpha). Par ailleurs, l'activation neutronique a été pendant de nombreuses années la technique de choix pour l'analyse d'ultra-traces, notamment pour les eaux [8]. Divers domaines ont stimulé l'amélioration des limites de détection soit d'éléments, soit d'isotopes mineurs de faible activité spécifique : la lutte contre la prolifération, les recherches relatives à la gestion des déchets nucléaires, et plus généralement les études d'impact de diverses installations nucléaires. Dans certains cas, l'analyse isotopique précise d'éléments présents eux-mêmes à l'état de traces s'est révélée nécessaire. En effet, certains isotopes mineurs d'actinides peuvent apporter des renseignements décisifs sur l'origine de certains composés suspects. De même, il est important de détecter les produits de fission à vie longue dont l'activité spécifique est très faible. Quelques grandes techniques sont actuellement au coude à coude pour ces analyses élémentaires ou isotopiques d'ultra-traces [9] (fig. 2). Trois d'entre elles reposent directement sur une détection par spectrométrie de masse : la spectrométrie de masse avec accélérateur (AMS) [10-14], le couplage torche à plasma/spectrométrie de masse (ICPMS) [15-17], et la spectrométrie de masse avec ionisation résonante (RIMS) $[18,19]$. Ces techniques permettent actuellement de détecter de l'ordrede $10^{6}-10^{8}$ atomes, 


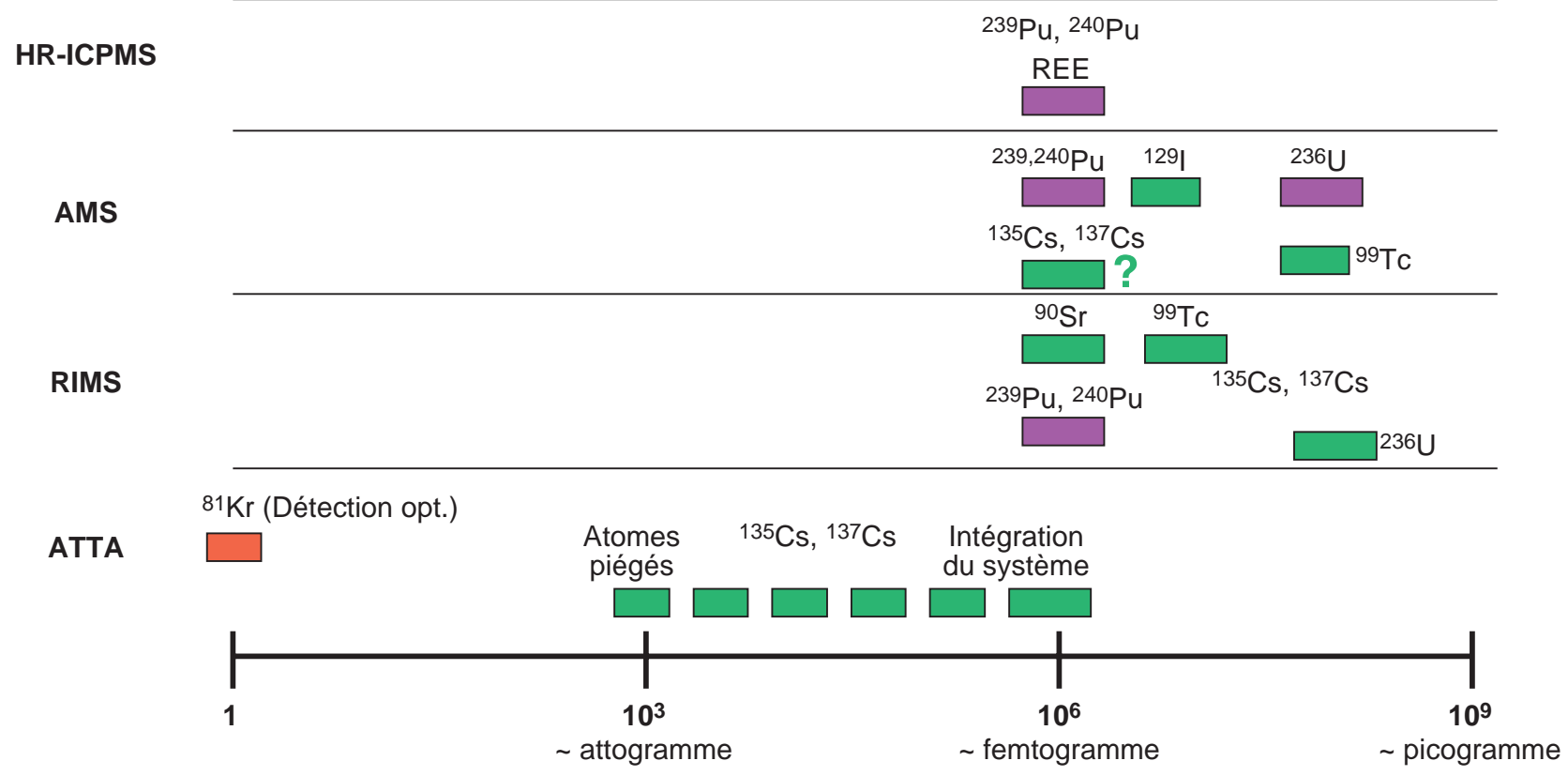

Figure 2

Performances comparées de méthodes pour la détection d'ultra-traces d'actinides et de produits de fission. Les quantités reportées sont en nombre d'atomes détectables (pour chaque isotope recherché), ou en masse .

HR-ICPMS : spectrométrie de masse à haute résolution couplée avec une torche à plasma. AMS : spectrométrie de masse par accélérateur. RIMS : spectrométrie de masse par ionisation résonante. ATTA : analyseur de traces par piégeage « d'atomes froids ».

Compared performances of various methods for the detection of trace amounts of actinides and fission products. Reported quantities are number of detected atoms (for each isotope).

HR-ICPMS: high resolution inductively coupled plasma mass spectrometry. AMS: accelarator mass spectrometry.

RIMS: resonant ionisation mass spectrometry. ATTA: atom trap trace analysis.

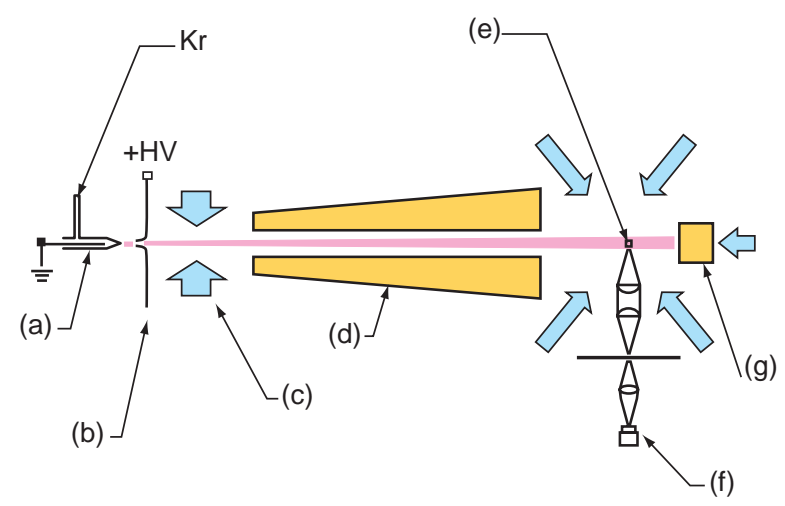

Figure 3

Principe du piégeage magnéto-optique utilisé à Argonne pour l'analyse d'ultra-traces. a) source à décharge ; b) skimmer ; c) refroidissement transverse ; d) ralentisseur Zeeman ; e) piège optique (lasers) ; f) détection des photons ; g) bobine d'équilibrage.

Principle ot the atom trap analysis system used in Argonne: a) discharge source; b) skimmer; c) transverse cooling; d) Zeeman slower; e) trap; f) photon detector; g) balance coil.). soit de 1 à 100 fg pour des éléments comme le $\mathrm{Pu}$, le Cs, ou l'iode. L'introduction d'une nouvelle technique (ATTA, Atom Trap Trace Analysis) exploitant le piégeage magnétooptique d'atomes froids, développée à Argonne [20, 21] et à Los Alamos [22, 23], puis actuellement au CEA Saclay [A. Petit, comm. pers.] permet d'atteindre la détection d'atomes uniques et de quantifier des rapports isotopiques ${ }^{135} \mathrm{Cs} /{ }^{137} \mathrm{Cs}$ sur quelques milliers d'atomes (fig. 3). Cette technique est actuellement parmi les plus sensibles que l'on puisse imaginer. On peut atteindre la mesure d'abondances isotopiques de $10^{-11}$ à $10^{-12}$, utiles pour la datation absolue d'eaux souterraines, utilisant la mesure du ${ }^{81} \mathrm{Kr}$ radiogénique, très peu abondant [21].

L'analyse élémentaire proprement dite a vu s'affronter les spectroscopies atomiques (absorption ou émission) et l'activation neutronique, longtemps incontournable pour la détection des ultra-traces. La multiplication des ICPMS a porté un coup presque fatal à l'activation neutronique, surtout compte tenu des contraintes de l'accès à un réacteur et à la manipulation d'échantillons activés. 


\section{SPÉCIATION EN PHASE LIQUIDE}

Simultanément, certaines spectroscopies ont permis, non seulement d'atteindre des limites de détection remarquables, mais aussi de fournir des informations sur la spéciation (tableau 1). La spéciation est une information essentielle à la prévision de la migration des radioéléments dans les milieux géologiques [24]. En ce qui concerne la spéciation en solution, trois approches sont possibles :

- la spéciation directe;

- la spéciation séquentielle;

- la spéciation par méthodes couplées.

La spéciation directe est obtenue par introduction directe de l'échantillon dans le système d'analyse, soit dilué, soit après addition de certains réactifs (qui ne doivent pas avoir d'influence sur la répartition des espèces en solution). C'est le cas pour les méthodes reposant sur la spectroscopie laser à résolution temporelle et la spectrométrie de masse avec injection directe dans une source de type FAB (Fast Atom Bombardment) ou électrospray. Ces méthodes sont rapides et permettent de dégrader le moins possible les caractéristiques de l'échantillon.

La spéciation séquentielle, qui est la méthode la plus ancienne repose sur des techniques séparatives (colonnes, résines) ou extractives (solvants) qui permettent de sélectionner les différentes espèces chimiques, qui sont ensuite quantifiées avec des techniques classiques d'analyse élémentaire.
La spéciation par méthodes couplées utilise deux appareils en série, l'un opérant la séparation chimique (HPLC, électrophorèse capillaire, etc.), l'autre permettant la détection élémentaire (ICP, ICPMS). Ces méthodes sont de plus en plus répandues et éprouvées, et permettent réellement de déterminer une spéciation sur des traces ou ultra-traces. Elles sont néanmoins assez lourdes et délicates à mettre en œuvre.

La spectrofluorimétrie laser à résolution temporelle (SLRT/TRLIF) a permis de descendre largement sous la ppt pour la mesure de l'uranium en solution [25], pour le curium [26]. Elle a également premis de déterminer la nature des complexes dans lesquels ces éléments (U, Cm, Eu) sont engagés ainsi que leur environnement, (fig. 4) [27, 28]. Cette méthode est élégante et sensible, mais elle reste tributaire du caractère fluorescent de certains éléments (uranium, terres rares, curium), elle n'est donc pas universellement applicable. Elle est concurrencée pour la spéciation par la spectrométrie de masse, de manière directe, dans une source de type électrospray [29], séparation préalable (HPLC) [30] ou électrophorèse capillaire $[31,32]$ puis transfert dans la source d'un ICPMS. En ce qui concerne les méthodes mettant en œuvre une introduction directe de l'échantillon dans la source d'un spectromètre de masse, il est possible de calculer la stabilité des espèces produites dans la source et de comparer avec les spectres de masse enregistrés. Ceci a été fait pour des métaux de transition [33,34], et semble maintenant possible pour des éléments plus lourds (4f ou 5f) [28]. Il est encore

\section{TABLEAU I}

Quelques méthodes permettant d'aborder la spéciation en solution des éléments au niveau des traces ou ultra-traces Liquid phase speciation methods for trace elements

Méthodes directes :

\begin{tabular}{l|l|l}
\hline \multicolumn{1}{c|}{ Méthode } & \multicolumn{1}{c}{ Principe } & Analyse quantitative \\
\hline SLRT/TRLIF & Fluorescence laser & Oui \\
Voltampérométrie & Méth. électrochimiques & Oui \\
ESI-MS & Spectrométrie de masse & à vérifier \\
SLPT & Lentille photo-thermique & Oui \\
Spectrophotométrie & Absorption UV-VIS & Oui, peu sensible \\
\hline
\end{tabular}

Méthodes séquentielles (+ analyse élémentaire par AA, ICP, ICPMS)

\begin{tabular}{l|l|l}
\hline \multicolumn{1}{c|}{ Méthode } & \multicolumn{1}{c}{ Principe } & Analyse quantitative \\
\hline SPE/AAS - ICP - ICPMS & Extraction support solide & Oui \\
ELL & Extraction liquide/liquide & Oui \\
REI & Résines échangeuses d'ions & Oui \\
\hline
\end{tabular}

Méthodes couplées

\begin{tabular}{l|l|l}
\hline \multicolumn{1}{c|}{ Méthode } & Principe & Analyse quantitative \\
\hline HPLC/ICP-MS & Chromatographie liquide & Oui \\
EC/ICP-MS & Électrophorèse capillaire & Oui \\
CI/ICP-MS & Chromatographie ionique & Oui \\
\hline
\end{tabular}


assez difficile de comparer les performances de ces méthodes en matière de spéciation sur des échantillons très faiblement concentrés. Les performances concernant l'analyse quantitative restent encore à confirmer. Les méthodes directes en spectrométrie de masse permettent de quantifier les rapports entre espèces. L'utilisation de méthodes de dilution isotopique pourrait permettre de quantifier les concentrations. En spectroscopie de fluorescence laser à résolution temporelle, la spéciation proprement dite est mesurable dans une gamme de $10^{-6}$ à $10^{-8} \mathrm{M}$, et permet une estimation quantitative de chaque espèce et de son niveau de concentration. Les méthodes couplées (HPLC, EPC) permettent généralement de descendre en dessous de la ppb pour les différentes espèces séparées. L'analyse quantitative est possible sur des échantillons un peu complexes, mais elle reste souvent handicapée par l'absence de matériaux de référence.

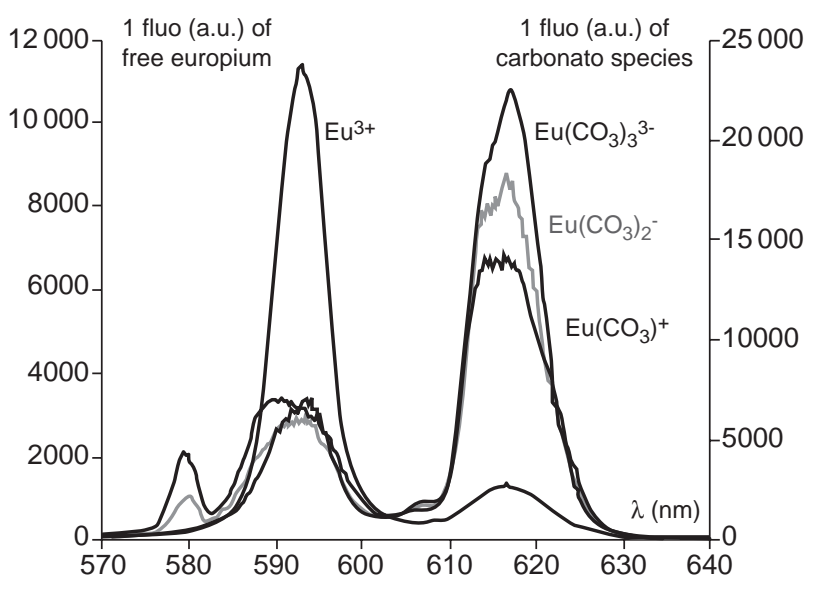

Figure 4

Spéciation directe de l'Eu en milieu carbonaté obtenue par spectrofluorimétrie laser à résolution temporelle.

Direct speciation of Eu in carbonate media obtained by time resolved laser induced fluorescence spectroscopy. From [27].

\section{SPÉCIATION DE SURFACE}

La mobilité des radioéléments ou des métaux lourds est conditionnée, non seulement par la ou les formes chimiques sous lesquelles ils sont présents en solution, mais aussi par la manière dont ils peuvent être piégés par adsorption et par coprécipitation sur les phases qu'ils sont susceptibles de rencontrer pendant leur parcours. Les oxydes de $\mathrm{Fe}$ ou de Ti, les argiles sont bien connus pour leur capacité à fixer les cations et certains anions (si le pH est compris entre 3 et 6). Pour autant, en conditions géologiques, il existe encore beaucoup d'incertitudes sur les mécanismes responsables de cette fixation. Les descriptions à l'échelle atomique et moléculaire sont encore très fragmentaires. Il est donc primordial d'identifier ces mécanismes, afin de fonder solidement les prévisions, et notamment les études d'impact. Dans le cas du stockage profond de déchets nucléaires en conditions géologiques, il apparaît que les éléments les plus radiotoxiques (actinides) sont complètement retenus, par adsorption ou précipitation, tandis que certains produits de fission à vie longue particulièrement solubles, comme l'iode 129, pourraient atteindre la biosphère après plusieurs millions d'années [24]. Le rôle de ces processus d'adsorption est donc primordial, en particulier pour les actinides, et il doit donc faire l'objet d'études appuyées sur la meilleure compréhension possible des phénomènes à l'échelle microscopique.

Ces études de spéciation de surface font appel à diverses techniques, généralement spectroscopiques (tableau 2). Parmi les méthodes employées, on peut citer la spectroscopie de photoélectrons, qui permet de différencier les degrés d'oxydation des espèces présentes en surface, mais aussi de voir un effet des liaisons chimiques. La spectrofluorimétrie laser à résolution temporelle est également utilisable et permet de différencier les signaux associés aux différentes espèces de surface envisageables [35-37]. Elle permet aussi d'analyser l'incorporation d'actinides dans des phases peu ou mal cristallisées [38]. La spectroscopie d'absorption X permet également d'obtenir des informations très précieuses sur l'environnement chimique de composés adsorbés, et de décrire les liaisons ainsi que la géométrie implicant les espèces de surface. Les études concernant les actinides [3941] et certains métaux homologues de produits de fission ou d'activation à vie longue [42] se multiplient.

Ces techniques ont permis de faire des progrès considérables, mais elles sont encore limitées à certains points de vue. Ainsi, la spectrofluorimétrie laser est toujours tributaire des propriétés de fluorescence, qui ne sont utilisables que pour quelques-uns des éléments $4 \mathrm{f}$ et $5 \mathrm{f}$ (voir supra). La spectroscopie de photoélectrons nécessite un vide très poussé, et il est possible que l'évolution sous vide des échantillons ne permette pas d'appréhender la réalité. La spectroscopie d'absorption des rayons $\mathrm{X}$ n'est pas limitée par ces problèmes d'ultra-vide, et permet de travailler avec des suspensions ou des solutions. Par contre, sa sensibilité est assez limitée et ne permet pas toujours d'approcher les conditions d'études d'une vraie sorption, où la concentration d'un élément susceptible de s'adsorber est très en deçà de sa limite de solubilité. Dans la mesure où certains supports adsorbants, en particulier les matériaux naturels, peuvent se révéler très hétérogènes, il est nécessaire d'obtenir une bonne résolution spatiale. Il se développe donc maintenant des lignes équipées de microfaisceaux $[40,43]$. 
TABLEAU 2

Méthodes utilisables pour obtenir des informations relatives à la spéciation de surface. Surface speciation methods for trace elements

\begin{tabular}{|c|c|c|c|}
\hline Méthode & Principe & Application & Sensibilité \\
\hline SLRT & $\begin{array}{l}\text { Fluorescence laser à résolution } \\
\text { temporelle sur suspensions }\end{array}$ & $\begin{array}{l}\text { Spectre influencé par l'environnement } \\
\text { chimique : spéciation }\end{array}$ & Bonne \\
\hline XPS & Spectroscopie de photoélectrons & $\begin{array}{l}\text { Degré d'oxydation, sensible à l'environnement } \\
\text { chimique, possibilité de résolution spatiale }\end{array}$ & Moyenne \\
\hline EXAFS & Spectroscopie d'absorption X & $\begin{array}{l}\text { Environnement chimique, résolution spatiale } \\
\text { si microfaisceau }\end{array}$ & Moyenne \\
\hline STXM & Imagerie $\mathrm{X}+$ absorption $\mathrm{X}$ au seuil du $\mathrm{C}$ & Imagerie + spéciation, résolution spatiale & Bonne \\
\hline IR ATD (p.m.) & $\begin{array}{l}\text { Spectroscopie de vibration } \\
\text { sur les espèces adsorbées }\end{array}$ & Spéciation & Moyenne à faible \\
\hline IBA & $\begin{array}{l}\text { Analyse par faisceau d'ions accélérés, } \\
\text { réactions nucléaires, photons } \\
\text { ou gamma induits }\end{array}$ & $\begin{array}{l}\text { Analyse élémentaire simultanée des éléments } \\
\text { lourds (PIXE) et des légers (RN), } \\
\text { résolution spatiale si microfaisceau }\end{array}$ & Bonne \\
\hline TOF-SIMS & $\begin{array}{l}\text { Spectrométrie de masse, } \\
\text { ions secondaires, mode statique }\end{array}$ & Spéciation, extrême surface, résolution spatiale & Bonne \\
\hline SNOM-MS & $\begin{array}{l}\text { Sonde champ proche ablation laser } \\
\text { et spectrométrie de masse }\end{array}$ & Spéciation, résolution spatiale de $100 \mathrm{~nm}$ & Bonne \\
\hline
\end{tabular}

Le développement de nouvelles lignes de lumière équipées de microfaisceaux permettant de faire de l'imagerie en mode balayage revêt un intérêt considérable. Outre la possibilité d'obtenir une image à très haute résolution, on peut aussi récupérer une information spectroscopique, pour une analyse élémentaire en exploitant la fluorescence X [44], et pour la spéciation, en utilisant l'adsorption $\mathrm{X}$ au seuil du $\mathrm{C}$ ou du S, qui permet de remonter à la nature des liaisons chimiques [45].

Les méthodes basées sur la spectrométrie de masse d'ions secondaires (SIMS) et notamment les nouvelles machines travaillant en statique, avec un analyseur en temps de vol (TOF-SIMS) offrent l'espérance d'une meilleure connaissance de la spéciation [46, 47]. En effet, l'ionisation plus « douce » permet de mieux préserver l'intégrité des molécules présentes en surface, et le mode de travail en statique, avec un courant d'ions secondaires très faible est quasiment non destructif. Cette méthode permet de faire de «l'imagerie moléculaire ».

Il est également possible d'obtenir des informations avec une excellente résolution spatiale en utilisant la technique SNOM (Scanning Near-Field Optical Microscopy). L'information spectroscopique locale (IR, Raman, Fluorescence) peut être exploitée [48]. Dans certains cas, il est possible de procéder à une nano-ablation de l'échantillon en utilisant la pointe optique $(100 \mathrm{~nm} \times 100 \mathrm{~nm})$ et de récupérer ce qui est ablaté pour l'introduire dans une source de spectromètre de masse [49]. Ce système, fonctionnant à pression atmosphérique, a été testé sur des systèmes modèles mais pourrait être développé avec profit pour des études environnementales.
Enfin, on peut constater que certaines techniques d'analyse basées sur l'utilisation de faisceaux d'ions accélérés, peuvent encore se révéler tout à fait concurrentielles pour la détection d'ultra-traces, notamment en mode microsonde [50].

On voit donc que la spéciation de surface peut être maintenant abordée avec de plus en plus de pertinence et de performances sur les trois volets suivants :

- sensibilité ;

- résolution spatiale;

- minimisation de la perturbation des échantillons (fonctionnement à pression atmosphérique).

Certaines de ces techniques peuvent permettre des analyses quantitatives, notamment les méthodes basées sur l'analyse par faisceaux d'ions accélérés. Dans certains cas, une bonne maîtrise des conditions expérimentales ainsi que la disponibilité d'étalons de composition et de structure proches des échantillons donnent accès à des analyses quantitatives.

\section{ANALYSE IN SITU VOIRE IN VIVO}

L'analyse in situ ou in vivo d'éléments à l'état de traces préoccupe de plus en plus d'équipes. En effet, il est maintenant essentiel de pouvoir récupérer l'information au cœur des systèmes environnementaux ou biologiques, sans les perturber. La mesure d'un potentiel redox ou d'un $\mathrm{pH}$ peut se révéler très difficile dans des fluides profonds, de même que pour certains éléments comme le Fe, indicateurs de l'état redox du milieu. De plus, le prélèvement perturbe l'équilibre de 
l'échantillon (oxydation, dégazage) et peut complètement altérer l'information attendue. Ainsi, l'oxydation très partielle d'une eau profonde réductrice provoque l'apparition de précipités d'hydroxydes ferriques qui vont piéger quasiment tous les métaux plurivalents à l'état de traces. La mesure d'éléments en traces in situ, la possibilité d'obtenir des informations en matière de spéciation sont des enjeux importants, qui commencent à trouver des solutions crédibles [2, 3]. Il y a loin du concept, ou du prototype de laboratoire, au système robuste et éprouvé, capable de fonctionner sur le terrain, a fortiori en forage profond. Certains dispositifs à base de capteurs optiques ont été développés pour la mesure du $\mathrm{pH}$, testés puis utilisés avec succès in situ ou en forage [51, 52]. Les méthodes électrochimiques sont aussi maintenant très abouties et permettent d'obtenir des performances très significatives pour une utilisation in situ [53]. L'analyse in vivo constitue un domaine très vaste, qui connaît de nombreux développements. Sans rentrer dans les détails, les méthodes optiques ou électrochimiques, parfois couplées, sont les plus aptes à permettre d'accéder au cœur des systèmes biologiques, sans les perturber, pour l'analyse de traces et ultra-traces en temps réel. Les applications des ultramicroélectrodes en sont l'un des meilleurs exemples [54].

\section{MINIATURISATION DES TECHNIQUES ET DES ÉCHANTILLONS}

Enfin, la miniaturisation des techniques, l'accès à des nanoéchantillons, la résolution latérale nanométrique annoncent la prochaine révolution de la chimie analytique (fig. 5). Les retombées de cette révolution seront immenses : accès au haut débit analytique à moindre coût, développement des systèmes analytiques embarqués, analyse en ligne, accès à des micromilieux (analyse directe de divers constituants de la cellule). Dans cette optique ont été lancées des études sur des microsystèmes analytiques, ( $\mu$-TAS, micro-Total Analytical Systems), qui permettant d'envisager des gains sur les aspects suivants :

- automatisation facile;

- consommation de réactifs divisée par $10^{4}$ à $10^{6}$;

- vitesse d'analyse multipliée par $10^{2}$;

- développement à grande échelle possible à bas coût ;

- systèmes jetables, peu onéreux.

Il est possible de décliner ce genre de concepts pour des analyses de traces métalliques, voire de spéciation. Les microsystèmes séparatifs (électrophorèse capillaire, électro-chromatographie) se perfectionnent. Les progrès de la
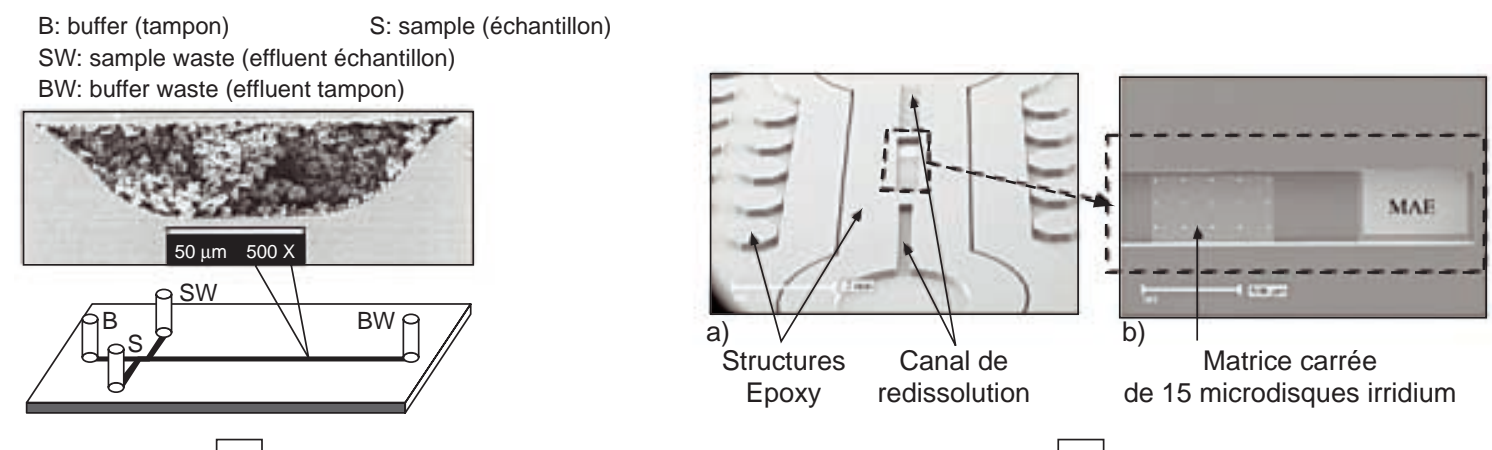

1

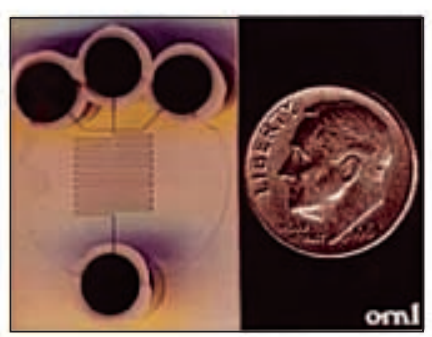

3

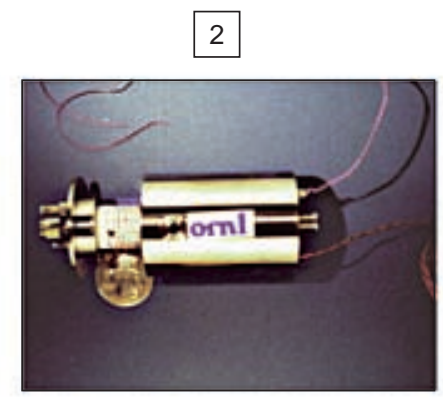

4

Figure 5

Exemples de microsystèmes analytiques.

1 : micro-électrochromatographie, phase stationnaire polymérisée in situ [55] ; 2 : microcellule voltammétrique utilisée pour l’analyse de traces métalliques [56]; 3 et $4:$ micro-électrophorèse capillaire et micro-spectromètre à mobilité ionique (IMS).

Example of analytical microsystems.

1: micro-electrochromatography: in situ polymerized stationary phase [55]; 2: microcell for voltammetry of trace metals [56]; 3 and 4: microcapillary electrophoresis and micro-ion mobility mass spectrometer. M.J. Ramsay, Oak Ridge National Laboratory (http://www.ornl.gov/sci/lsm/projects1.html, http://www.ornl.gov/sci/eere/PDFs/IMS_Factsheet.pdf) 
microfluidique permettent d'envisager des dérivations. Les méthodes de détection commencent à se miniaturiser également, soit sous forme de détection classique en absorbance UV-VIS, soit en fluorescence laser, soit en utilisant d'autres techniques (lentille photothermique). La détection peut aussi s'envisager par spectrométrie de masse, les spectromètres de masse eux-mêmes sont actuellement en voie de miniaturisation extrême. Enfin, les méthodes de détection électrochimiques sont aussi très adaptées à une miniaturisation, et par nature, faciles à intégrer à des microcircuits. Ainsi, l'équipe de J. Buffle, de l'université de Genève a mis au point, avec des collègues de l'IMT de Neuchâtel un système miniaturisé de capteur électrochimique pour les métaux lourds, fonctionnant en voltammétrie cyclique, permettant de détecter quelques picomoles pour le plomb, et quelques dizaines de picomoles pour le cadmium. Des informations relatives à la spéciation peuvent être également obtenues [56].

Pour le monde nucléaire, la systématisation de nanoéchantillons et de méthodes capables de les prendre en compte permet d'espérer s'affranchir rapidement des contraintes traditionnelles liées à l'analyse d'échantillons radioactifs. On peut ainsi entrevoir la manipulation de quantités d'actinides ou de produits de fission présentant un niveau d'activité compatible avec une manipulation hors boîte à gant. Les effluents analytiques générés seront également très réduits, ce qui permettra d'abaisser fortement les coûts.

D'une manière générale, l'introduction de ces microsystèmes - une fois leur maturité technique établie — sur des ensembles d'analyse en ligne, permet d'envisager une révolution dans les 5 à 10 ans à venir concernant les analyses de procédés, mais aussi les analyses environnementales. Il est même possible que la chimie analytique dans son ensemble puisse connaître une révolution comparable à celle de la microélectronique au début des années 1960.

\section{CONCLUSION}

En conclusion, il est primordial de rappeler que ces progrès en matière de quantité analysable ou de limite de détection nécessitent des exigences accrues quant au processus de prélèvement et à la représentativité de l'échantillon. Quiconque a travaillé sur des traces et surtout ultra-traces connaît les difficultés de maitriser les blancs... L'analyste, pour des sujets et des systèmes aussi complexes, doit être impliqué dans le questionnement, et ce, dès le départ. Tout ceci plaide pour une intégration de plus en plus poussée de l'analyse au sein des projets de recherche ou de développement, que ce soit en début de projet (que peut-on mesurer ?), en cours de projet (comment prélever et mesurer ?), ou en fin de projet (comment interpréter ?). Les techniques progressent vite et atteignent des performances inimaginables il y a encore dix ans. Celles qui se développent, et dépassent le stade du prototype de paillasse, sont souvent celles qui ont su répondre dès le début aux bonnes questions, qui ont su s'adapter aux laboratoires ou au terrain, et qui ont surtout permis de progresser dans l'interprétation des phénomènes étudiés, ou d'améliorer significativement les procédés.

En matière de spéciation, les besoins sont divers. Quand on aborde la réactivité chimique d'un métal dans son environnement, il est important de connaître la répartition des différentes formes chimiques (pourcentage de chaque espèce). Il n'est pas toujours utile ou significatif d'avoir simultanément l'analyse quantitative de l'élément. Certaines méthodes permettent d'avoir les deux types d'information, et d'autres, ne donnent accès qu'à l'une d'entre elles.

Le dernier message important concerne la révolution annoncée dans l'analyse de traces et ultra-traces grâce à l'arrivée des microsystèmes. L'évolution des coûts, la diminution des délais, l'augmentation des débits, les perspectives pour l'analyse in situ ou le contrôle en ligne permettront à un très grand nombre d'équipes d'avoir accès directement à l'analyse de traces ou ultra-traces dans des systèmes complexes.

\section{REFERENCES}

1 Cyanide Spill at Baia Mare, Romania (2000) UNEP/OCHA Assessment Mission, Rapport, 23 février-6 mars, http://www.tisaforum.org.yu/arhiva/BMFinRep.pdf.

2 Ben Ali M., Lemiti, M., Jaffrezic-Renault, N., Martelet, C., Chovelon, J.M. et Ben Ouada, H. (2001) Thin film microfabrication of gold microelectrodes functionalized with thiacalix[4]arene layer: applications to copper ion sensor. Thin Solid Films, 383, 292-295.

3 Saban, S.B. et Darling, R.B. (1999) Multi-element heavy metal ion sensors for aqueous solutions. Sensors and Actuators, B: Chemical, B61, 1-3, 128-137.

4 Ueberfeld, J., Parthasarathy, N., Zbinden, H., Gisin, N. et Buffle, J. (2002) Coupling Fiber Optics to a Permeation Liquid Membrane for Heavy Metal Sensor Development. Analytical Chemistry, 74, 3, 664-670.

5 Toulhoat, P. (1993) Application des capteurs à fibres optiques en sciences de la terre. Géologues, 99, 43-45.

6 Herzig, R., Rehnert, A., Korhammer, S., Kumpulainen, J., Schramel, P., Muntau, H., Linsinger, T. et Quevauviller, P. (2002) Certification of a new cabbage reference material for the quality control of trace-element determinations with some considerations on moisture. TrAC, Trends in Analytical Chemistry, 21, 11, 746-761.

7 Cornelis, R., Crews, H., Donard, O.F.X., Ebdon, L. et Quevauviller, Ph. (2001) Trends in certified reference materials for the speciation of trace elements. Fresenius, Journal of Analytical Chemistry, 370, 2-3, 120-125.

8 Alaux-Negrel, G., Beaucaire, C., Michard, G., Toulhoat, P. et Ouzounian, G. (1993) Trace-metal behavior in natural granitic waters. Journal of Contaminant Hydrology, 13, 1-4, 309-25.

9 Toulhoat, P. (2003) New frontiers in analytical chemistry of actinides and fission products. $9^{\text {th }}$ International Conference on the Chemistry and Migration Behaviour of Actinides and Fission Products in the Geosphere, Gyeongju, Corée, 20-26 septembre. 
10 McAninch, J.E., Hamilton, T F., Brown, T.A., Jokela, T.A., Knezovich, J.P., Ognibene, T. J., Proctor, I.D.; Roberts, M.L. Sideras-Haddad, E.; Southon, J.R. et Vogel, J.S. (2000) Plutonium measurements by accelerator mass spectrometry at LLNL. Nuclear Instruments \& Methods in Physics Research, Section B: Beam Interactions with Materials and Atoms, 172, 711-716.

11 Bergquist, B.A., Marchetti, A.A., Martinelli, R.E., McAninch, J.E., Nimz, G J.; Proctor, I. D., Southon, J.R. et Vogel, J.S. (2000) Technetium measurements by accelerator mass spectrometry at LLNL. Nuclear Instruments \& Methods in Physics Research, Section B: Beam Interactions with Materials and Atoms, 172, 328-332.

12 Beninson, D,. D’Amatto, E., Oliveira, A.A., Stark, J.W., Bonino, N.O., Bustos, G.R., Alvarez, D.E., Amodei, A.J., Bonino, A.G., Giannico, M.A. et Pomar, C. (2000) New dedicated AMS system at the Nuclear Regulatory Authority in Argentina. Nuclear Instruments \& Methods in Physics Research, Section B: Beam Interactions with Materials and Atoms, 172, 24-28.

13 Fifield, L.K. (2000) Advances in accelerator mass spectrometry. Nuclear Instruments \& Methods in Physics Research, Section B: Beam Interactions with Materials and Atoms, 172, 134-143.

14 Berkovits, D., Feldstein, H., Ghelberg, S., Hershkowitz, A., Navon, E. et Paul, M. (2000) ${ }^{236} \mathrm{U}$ in uranium minerals and standards. Nuclear Instruments \& Methods in Physics Research, Section B: Beam Interactions with Materials and Atoms, 172, 372-376.

15 Kim, C.S. et Kim, C.K. (2002) Determination of Pu sotopes in seawater by an on-line sequential injection technique with sector field inductively coupled plasma mass spectrometry. Anal. Chem., 74, 3824-3832.

16 Field, M.P. et Sherrell, R.M. (1998) Magnetic Sector ICPMS with desolvating micronebulization: interference-free subpicogram determination of rare earth elements in natural samples. Analytical Chemistry, 70, 21, 4480-4486.

17 Becker J.S. (2002) ICP-MS Determination of long-lived radionuclides. Spectroscopy Europe, 14/6, 8-16.

18 Gruning, C., Huber, G., Kratz, J.V., Passler, G., Trautmann, N., Waldek, A. et Wendt, K. (2001). Determination of trace amounts of plutonium in environmental samples by RIMS using a high repetition rate solid state laser system. AIP Conference Proceedings, 584 (Resonance Ionization Spectroscopy 2000), 255-260.

19 Wendt, K., Trautmann, N. et Bushaw, B.A. (2000) Resonant laser ionization mass spectrometry: an alternative to AMS? Nuclear Instruments \& Methods in Physics Research, Section B: Beam Interactions with Materials and Atoms, 172, 162169.

20 Bailey, K., Chen, C.Y., Du, X., Li, Y.M., Lu, Z.T., O'Connor, T.P. et Young, L. (2000) ATTA - A new method of ultrasensitive isotope trace analysis. Nuclear Instruments \& Methods in Physics Research, Section B: Beam Interactions with Materials and Atoms, 172, 224-227.

21 Bailey, K., Chen, C.Y., Du, X., Li, Y.M., Lu, Z.T., O'Connor, T.P. et Young, L. (2000) Atom trap trace analysis. Hyperfine Interactions, 127, 1-4, 515-518.

22 Vieira, D.J., Crane, S.G., Guckert, R. et Zhao, X. (2000) Trapping radioactive atoms for basic and applied research. Hyperfine Interactions, 127, 1-4, 387-393.

23 Di Rosa, M.D., Crane, S.G., Kitten, J J., Taylor, W.A., Vieira, D.J. et Zhao, X. (2003) Magneto-optical trap and mass-separator system for the ultra-sensitive detection of ${ }^{135} \mathrm{Cs}$ and ${ }^{137} \mathrm{Cs}$. Applied Physics B: Lasers and Optics, 76, 1 , 45-55.
24 Toulhoat, P. (2002) Confinement and migration of radionuclides in a nuclear waste deep repository. Comptes rendus Physique, 3, 7-8, 975-986.

25 Moulin, C. Beaucaire, C. Decambox, P. Mauchien, P. (1990) Determination of uranium in solution at the ng L-1 level by time-resolved laser-induced spectrofluorometry: application to geological samples. Analytica Chimica Acta, 238, 2, 291-6.

26 Moulin, C., Decambox, P. et Mauchien, P. (1991) Determination of curium at $0.1 \mathrm{ng} \mathrm{L}-1$ levels in a micellar medium by time-resolved laser-induced spectrofluorometry. Analytica Chimica Acta, 254,1-2, 145-51.

27 Plancque, G ., Moulin, V., Toulhoat, P,; Moulin, C. (2003) Europium speciation by time-resolved laser-induced fluorescence. Analytica Chimica Acta, 478, 1, 11-22.

28 Stumpf, T., Fanghaenel, T. et Grenthe, I. (2002) Complexation of trivalent actinide and lanthanide ions by glycolic acid: a TRLFS study. Journal of the Chemical Society, Dalton Transactions, 20, 3799-3804.

29 Plancque, G., Moulin, V., Amekraz, B., Moulin, C., Toulhoat, P. (2000) Speciation of radionuclides by electrospray ionisation mass spectrometry. $15^{\text {th }}$ IMSC International Mass Spectrometry Conference, Barcelone, Espagne.

30 Betti, M., Aldave de las Heras, L. Perna, L., Bocci, F. et Huber, T. (2002) Hyphenated techniques for speciation of uranium and other actinides. Uranium in the Aquatic Environment, Proceedings of the International Conference [on] Uranium Mining and Hydrogeology III and the International Mine Water Association Symposium, Freiberg, Allemagne, 15-21 sept., 377-384.

31 Kuczewski, B., Marquardt, C.M., Seibert, A., Geckeis, H.; Kratz, J.V. et Trautmann, N (2003) Separation of plutonium and neptunium species by capillary electrophoresisinductively coupled plasma-mass spectrometry and application to natural groundwater samples. Analytical Chemistry, 75, 24, 6769-6774.

32 Baglan, N., Delorme, A. et Aupiais, J. (2003) Interface development for coupling Capillary Electrophoresis (CE) and Inductively Coupled Plasma-Mass Spectrometry (ICP-MS). Application to plutonium speciation. AIP Conference Proceedings, 673 (Plutonium Futures -The Science), 393395.

33 Boutreau, L., Leon, E., Rodriguez-Santiago, L., Toulhoat, P., Mo, O. et Tortajada, J. (2002) Gas-phase reactivity of $\mathrm{Cu}^{+}$ and $\mathrm{Ag}^{+}$with glycerol: an experimental and theoretical study. Journal of Physical Chemistry, A, 106, 44, 10563-10577.

34 Boutreau, L., Toulhoat, P., Tortajada, J, Luna, A., Mo, O. et Yanez, M. (2002) Reactions between glycolic acid and $\mathrm{Cu}^{+}$ in the gas phase. An Experimental and theoretical study. Journal of Physical Chemistry, A, 106, 40, 9359-9368.

35 Drot, R., Simoni, E., Alnot, M. et Ehrhardt, J.J. (1998) Structural environment of uranium(VI) and europium(III) species sorbed onto phosphate surfaces: XPS and optical spectroscopy studies. Journal of Colloid and Interface Science, 205, 2, 410-416.

36 Alliot, C. (2003) Sorption de lanthanides et actinides en présence de complexants organiques simples. Approche thermodynamique et spectroscopique. Thèse de doctorat, université d'Évry-Val d'Essonne.

37 Kowal-Fouchard, A., Drot, R., Simoni, E. et Ehrhardt, J.J. (2004) Use of spectroscopic techniques for uranium(VI)/montmorillonite interaction modeling. Environmental Science and Technology, 38, 5, 1399-1407.

38 Tits, J., Stumpf, T., Rabung, T., Wieland, E. et Fanghaenel, T. (2003) Uptake of Cm(III) and Eu(III) by calcium silicate hydrates: a solution chemistry and time-resolved laser 
fluorescence spectroscopy study. Environmental Science and Technology, 37, 16, 3568-3573.

39 Reich, T., Moll, H., Arnold, T., Denecke, M.A., Hennig, C., Geipel, G., Bernhard, G., Nitsche, H., Allen, P.G., Bucher, J.J., Edelstein, N.M. et Shuh, D.K. (1998) An EXAFS study of uranium(VI) sorption onto silica gel and ferrihydrite. Journal of Electron Spectroscopy and Related Phenomena, 96, 1-3, 237-243.

40 Duff, M.C., Newville, M., Hunter, D.B., Bertsch, P.M., Sutton, S.R., Triay, I.R., Vaniman, D.T., Eng, P. et Rivers, M.L. (1999) Micro-XAS studies with sorbed plutonium on tuff. Journal of Synchrotron Radiation, 6, 3, 350-352.

41 Daehn, R., Scheidegger, A.M.; Manceau, A., Curti, E., Baeyens, B., Bradbury, M.H. et Chateigner, D. (2002) Th uptake on montmorillonite: a powder and polarized extended X-ray absorption fine structure (EXAFS) study. Journal of Colloid and Interface Science, 249, 1, 8-21.

42 Daehn, R., Scheidegger, A.M., Manceau, A., Schlegel, M.L., Baeyens, B., Bradbury, M. H. et Chateigner, D. (2003) Structural evidence for the sorption of $\mathrm{Ni}(\mathrm{II})$ atoms on the edges of montmorillonite clay minerals: a polarized X-ray absorption fine structure study. Geochimica et Cosmochimica Acta, 67, 1, 1-15.

43 Scheidegger, A., Grolimund, D. et Daehn, R. (2003) Use of microXAS to study the influence of the inherent heterogeneity of nuclear-waste repository materials on radionuclide uptake. Abstracts of Papers, 225th ACS National Meeting, New Orleans, LA, Etats-Unis, 23-27 mars.

44 Neuhausler, U., Schmidt, C., Hoch, M. et Susini, J. (2003) Fluorescence X-ray microscopy on hydrated tributyltin-clay mineral suspensions. Journal de Physique IV: Proceedings, 104 (X-Ray Microscopy), 443-446.

45 Plaschke, M., Rothe, J., Schafer, T., Denecke, M.A., Dardenne, K., Pompe, S. et Heise, K.H. (2002) Combined AFM and STXM in situ study of the influence of Eu(III) on the agglomeration of humic acid. Colloids and Surfaces, A: Physicochemical and Engineering Aspects, 197, 1-3, 245-256.

46 Stipp, S.L.S., Hansen, M., Kristensen, R., Hochella, M.F., Bennedsen, L., Dideriksen, K., Balic-Zunic, T., Leonard, D. et Mathieu, H.J. (2002) Behaviour of Fe-oxides relevant to contaminant uptake in the environment. Chemical Geology, 190, 1-4, 321-337.

47 Van Ham, R., Adriaens, A., Van Vaeck, L., Gijbels, R. et Adams, F. (2000) Molecular information in static SIMS for the speciation of inorganic compounds. Nuclear Instruments
\& Methods in Physics Research, Section B: Beam Interactions with Materials and Atoms, 161-163, 245-249.

48 De Serio, M., Zenobi, R. et Deckert, V. (2003) Looking at the nanoscale: scanning near-field optical microscopy. $\operatorname{Tr} A C$, Trends in Analytical Chemistry, 22, 2, 70-77.

49 Stoeckle, R., Setz, P., Deckert, V., Lippert, T., Wokaun, A. et Zenobi, R. (2001) Nanoscale atmospheric pressure laser ablation-mass spectrometry. Analytical Chemistry, 73, 7, 1399-1402.

50 Mercier, F., Moulin, V., Barre N., Trocellier, P. et Toulhoat, P. (2001) Study of A Ternary System Silica/Humic Acids/Iodine: Capabilities of the Nuclear Microprobe Nuclear Instruments and Methods in Physics Research, B 181, 628-633.

51 Duréault, B., Michels, M.H., Motellier S. et Toulhoat, P. (1993) Fiber optic pH sensor for in-situ applications. Sensors and Actuators, B 11, 467-473.

52 Beaucaire, C., Pitsch, H. et Toulhoat, P. (1995) Chemical characterisation and modelling of the interstitial fluid in the boom clay formation. 8th International Symposium on Water/Rock Interaction - WRI8, Proceedings, Y. Kharaka and O. Chudaev (éds), Balkema Rotterdam, 779-782.

53 Tercier-Waeber, M.L., Buffle, J., Koudelka-Hep, M. et Graziottin, F. (2002) Submersible voltammetric probes for in situ real-time trace element monitoring in natural aquatic systems, Ch. 2. In: Environmental Electrochemistry: Analysis of Trace Element Biogeochemistry, M. Taillefert, T.F. Rozan (éds.), American Chemical Society, Symposium Series, 811, Washington DC.

54 Amatore, C., Bouret, Y. et Midrier, L. (1999) Time resolved dynamics of the vesicle membrane during individual exocytotic secretion events as extracted from amperometric monitoring of adrenaline exocytosis by chromaffin cells. Chem. Eur. J., 5, 1999, 2151-2162.

55 Throckmorton D.J., Shepodd T.J. et Singh A.K. (2002) Electrochromatography in microchips: Reversed-phase separation of peptides and amino-acids using photo-patterned rigid polymer monolights. Anal. Chem., 74, 4, 784-789.

56 Salaun, P., Guenat, O., Berdondini, L., Buffle J. et KoudelkaHep, M. (2003) Voltammetric microsystem for trace elements monitoring. Analytical Letters, 36-9, 1835-1849.

Manuscrit définitif reçu en mars 2005 or distributed for profit or commercial advantage and that copies bear this notice and the full citation on the first page. Copyrights for components of this work owned by others than IFP must be honored. Abstracting with credit is permitted. To copy otherwise, to republish, to post on servers, or to redistribute to lists, requires prior specific permission and/or a fee: Request permission from Documentation, Institut français du pétrole, fax. +33147527078 , or revueogst@ifp.fr. 